\title{
MicroRNA clustering on the biogenesis of suboptimal microRNAs
}

\author{
Sehee Park' , Igojo Kang ${ }^{2}$ and Chanseok Shin $2,3,4,5^{*}$
}

\begin{abstract}
Most microRNAs (miRNAs) are processed by two ribonuclease III enzymes. The first cleavage is performed by Microprocessor that is composed of RNase III enzyme Drosha and DGCR8, and the second by another RNase III enzyme Dicer. There are many examples of miRNAs that are poor substrates for Drosha and Dicer, owing to their suboptimal structures. However, a number of these suboptimal miRNAs are known to be expressed at the same or higher level as their neighboring structurally-optimal miRNAs. Recent studies suggest that the clustered orientation of these suboptimal miRNAs is the explanation for this phenomenon. It has been observed that the biogenesis of these suboptimal miRNAs can be affected by the expression of their neighboring optimal miRNAs. This principle is expected to apply more broadly, as it has been shown that a large percentage of suboptimal miRNAs reside within operons.
\end{abstract}

Keywords: miRNA cluster, Pri-miR-144 451, Suboptimal structure, Non-canonical biogenesis, Microprocessor

\section{Biogenesis of canonical and non-canonical microRNAs}

MicroRNAs (miRNAs) are small, non-coding RNAs comprising $\sim 22$ nucleotides that play important roles in gene regulation $[1,2]$. miRNAs form a silencing complex with Argonaute protein (AGO) and together direct the posttranscriptional repression of mRNAs. After one strand of miRNA duplex is loaded into AGO, an miRNA seeks out and pairs with the target mRNA, which is then repressed by AGO [3].

In the canonical pathway of miRNA biogenesis, a long primary miRNA transcript (pri-miRNA) undergoes two cleavages (Fig. 1A). Pri-miRNAs are generated via the transcription of miRNA genes by RNA polymerase II (RNAPII), and they carry distinct stem-loop structures that are necessary for both of the cleavage reactions [4]. The first cleavage is performed by the Microprocessor complex, which consists of Drosha, a member of RNase III family, and two molecules of its cofactor, DGCR8 [5]. The Microprocessor recognizes and binds to the distinct

\footnotetext{
*Correspondence: cshin@snu.ac.kr

2 Department of Agricultural Biotechnology, Seoul National University,

Seoul 08826, Republic of Korea

Full list of author information is available at the end of the article
}

stem-loop structure of a pri-miRNA and cleaves the hairpin at about one helical turn from its base, releasing the precursor miRNA (pre-miRNA) [6]. This pre-miRNA is then exported to the cytoplasm, where a second cleavage is performed by Dicer, another RNase-III-type endonuclease [7]. Similar to Drosha, Dicer recognizes certain structures of pre-miRNAs rather than being sequence specific. Dicer cleaves the pre-miRNA at about two helical turns away from its base and releases an miRNA duplex. As mentioned earlier, one strand of this miRNA is then loaded into AGO to form a silencing complex that regulates mRNA expression [8].

Along with this canonical pathway of miRNA biogenesis, there are also numerous variations, in which miRNA substrates undergo non-canonical biogenesis (Fig. 1B). A number of Drosha-independent and Dicer-independent miRNA biogenesis pathways have been reported [9-12]. For example, "mirtrons" that are introns that mimic the structural features of pre-miRNAs and enter the miRNA biogenesis pathway have been identified [10]. It also has been found that some pre-miRNAs can be derived from tRNA genes without being processed by Drosha [11]. Despite these examples of miRNAs that bypass Drosha processing, there 


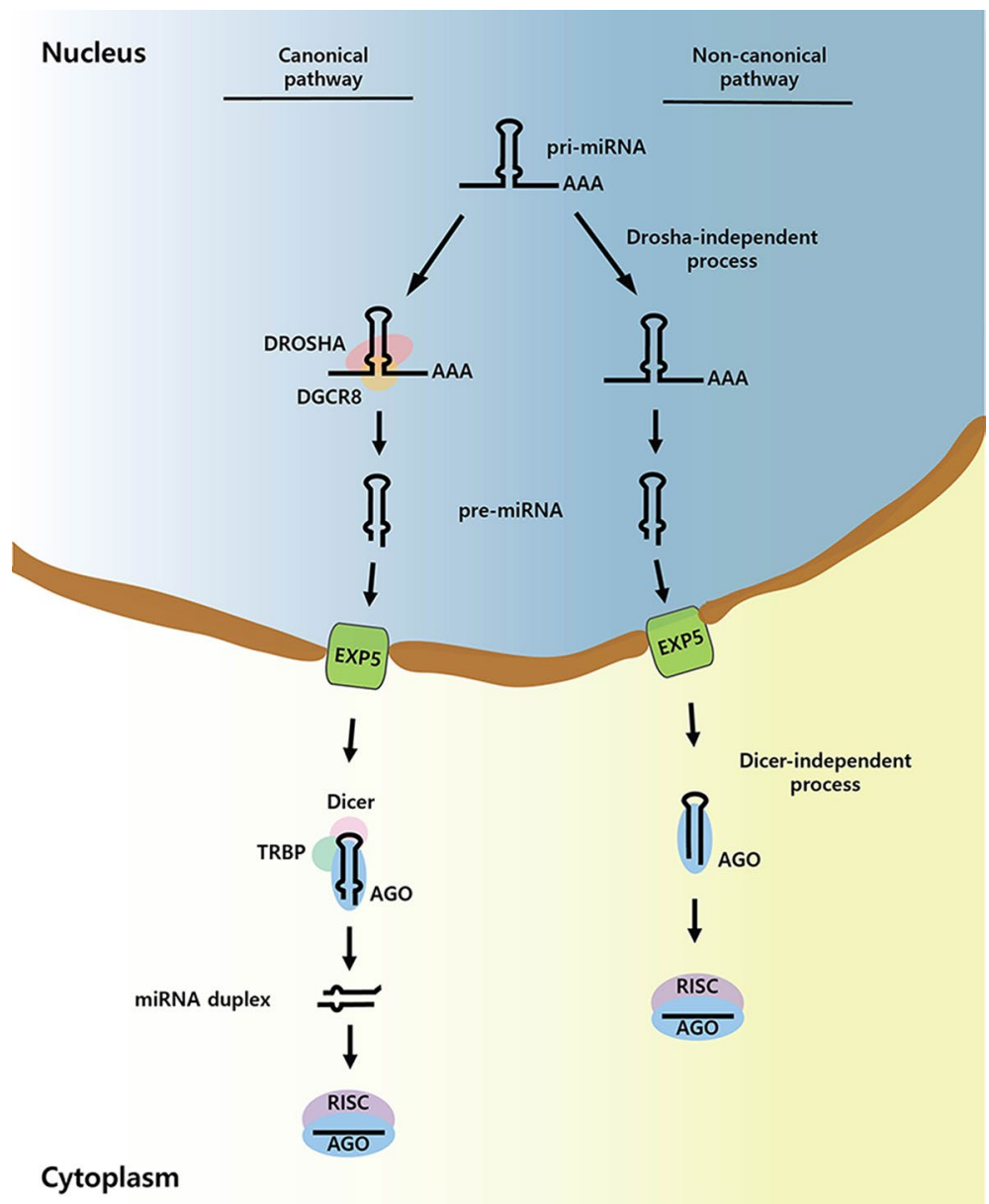

Fig. 1 Canonical and non-canonical pathways of miRNA biogenesis. A Canonical pathway of miRNA biogenesis. Primary transcripts (pri-miRNAs) are transcribed from miRNA genes by RNA polymerase II and are then cleaved by Microprocessor (Drosha+DGCR8) to form precursor miRNAs (pre-miRNAs). Pre-miRNAs are then exported to the cytoplasm, where they are once again cleaved by Dicer, forming miRNA duplexes. One strand of this miRNA duplex is loaded into Argonaute protein (AGO) to form RISC, which takes part in mRNA regulation. B Non-canonical pathway of miRNA biogenesis. During the non-canonical pathway of miRNA biogenesis, miRNAs bypass the Drosha- or Dicer-dependent cleavage step, and this step is replaced by another cleavage reaction carried out by different proteins

is only one known example of a miRNA that is Dicerindependent: miR-451 [12]. Therefore, many studies have been conducted to study the biogenesis pathway of miR-451, and numerous unique characteristics of this miRNA have been discovered recently [13-16].

Including miR-451, around one-third of vertebrae miRNAs exist in operons [17], and around 50\% of conserved miRNA genes in the human genome are clustered together [18]. Although the biological implications of this clustering are still mostly unclear, many recent studies have suggested that the clustering of miRNAs provide an advantage during miRNA biogenesis [13, 15, 16, 19]. More specifically, this clustered arrangement may allow pri-miRNAs that are structurally poor substrates of Microprocessor to be efficiently processed, allowing them to successfully enter the miRNA biogenesis pathway.

Recently, many studies have been conducted on miRNAs that are present on the same primary transcripts, for which the expression of one of the miRNAs is dependent on that of the other $[13,15,16,19]$. This phenomenon is referred to as "cluster assistance" [13].

\section{MicroRNA expression can be affected by neighboring clustered miRNA in cis Biogenesis of Drosophila miR-998 is dependent on neighboring miR-11}

Two miRNAs within the Drosophila E2f1 gene, miR-11 and miR-998, comprise the mir-11 998 cluster. Although both miR-11 and miR-998 are known to be co-expressed 
within the host gene, it has recently been reported that the expression of miR-998 is strongly dependent on the existence of the neighboring miR-11 gene [20]. This dependency was found by comparing the results of quantitative RT-PCR and Northern blots of wild-type miR998 and miR-11, reciprocal mutant alleles, the miR-11 deletion $(m R r-11 \Delta 1)$, and an $m i R-998$ mutant allele with imprecise $P$-element excision (miR-998 $\left.{ }^{\text {exc } 222}\right)[21,22]$. As a result, miR-11 in both wild-type and the miR-998exc222 was expressed at the usual level whereas the expression of miR-11 in miR-11 1 was absent. The expression of miR-11 was reduced in the miR-1141/miR-998 exc222 heterozygote.

On comparing these results with the expression of miR-998 in each mutant, miR-998 expression was not observed in either qRT-PCR or Northern blot analysis of $m i R-11 \Delta 1$. Furthermore, considering the results of $m i R$ $11 \Delta 1 /$ miR-998 $8^{\text {exc222 }}$, the expression of mir-11 in trans did not retrieve the expression of miR-998. These data suggest that the existence of miR-11 within the cluster is required for the expression of miR-998 [20].

To investigate the mechanism of this regulation, a primary miR-11 998 transcript was inserted downstream of the luciferase gene in the $3^{\prime}$ UTR [20]. If the pri-miRNA is cleaved by Microprocessor, then the luciferase transcript will be degraded that could be detected by measuring the decrease in luciferase activity. As a result, the decrease in luciferase activity was detected in the wild-type primiR-11 998. However, in the pri-miR-11 11 998 transcript, luciferase activity was maintained at the same level [20]. The results of this experiment suggest a mechanism in which the presence of $m i R-11$ is necessary for miR-998 to be successfully processed by Drosha.

\section{Biogenesis of Epstein-Barr virus miR-BHRF1-3 is dependent on neighboring miR-BHRF1-2}

Regulation among clustered miRNA genes has also been observed in viral miRNA clusters. The BHRF1 miRNA cluster in Epstein-Barr virus (EBV) consists of three genes: miR-BHRF1-1, miR-BHRF1-2, and miR-BHRF1-3. The co-expression of all three genes is required for the EBV virus to transform the resting B cells [23]. The efficiency of this viral transformation drops by about 20 -fold if the BHRF1 miRNAs are not present. This significant decrease in efficiency is mainly caused by miR-BHRF1-2 and miR-BHRF1-3. It has been observed that a virus without pre-miR-BHRF1-2 shows a decrease in the level of expression of miR-BHRF1-3 [24, 25], as demonstrated by investigating the downregulation of miR-BHRF1-3 in the miR-BHRF1-2 deleted $(\Delta 2)$ mutant. Both the $\Delta 2$ virus and the wild-type sequence were cloned into a eukaryotic expression plasmid and the expression levels of BHRF1 miRNA were measured. The results obtained from these cloned plasmids showed that the expression of miR-BHRF1-3 is highly dependent on the existence of miR-BHRF1-2, a finding that is in line with the results obtained from the $\Delta 2$ mutant virus, indicating that this expression pattern depends only on the genetic elements of the BHRF1 locus [24].

\section{Biogenesis of miR-497a is dependent on neighboring miR-195a}

CRISPR/Cas9 technology has been used to discover numerous other examples of gene regulation in clustered miRNA genes, including cluster miR-497 195. This cluster is composed of two miRNAs: miR-497a and miR-195a [26]. By targeting the hairpin structure of miR-195a with CRISPR/Cas9, the expression of miR-195a, as measured using qPCR, was downregulated by $55 \%$, and this downregulation eventually led to a significant decrease in the expression level of miR-497a as well [27]. As no mutation was detected in the sequence of miR-197a, it appears that

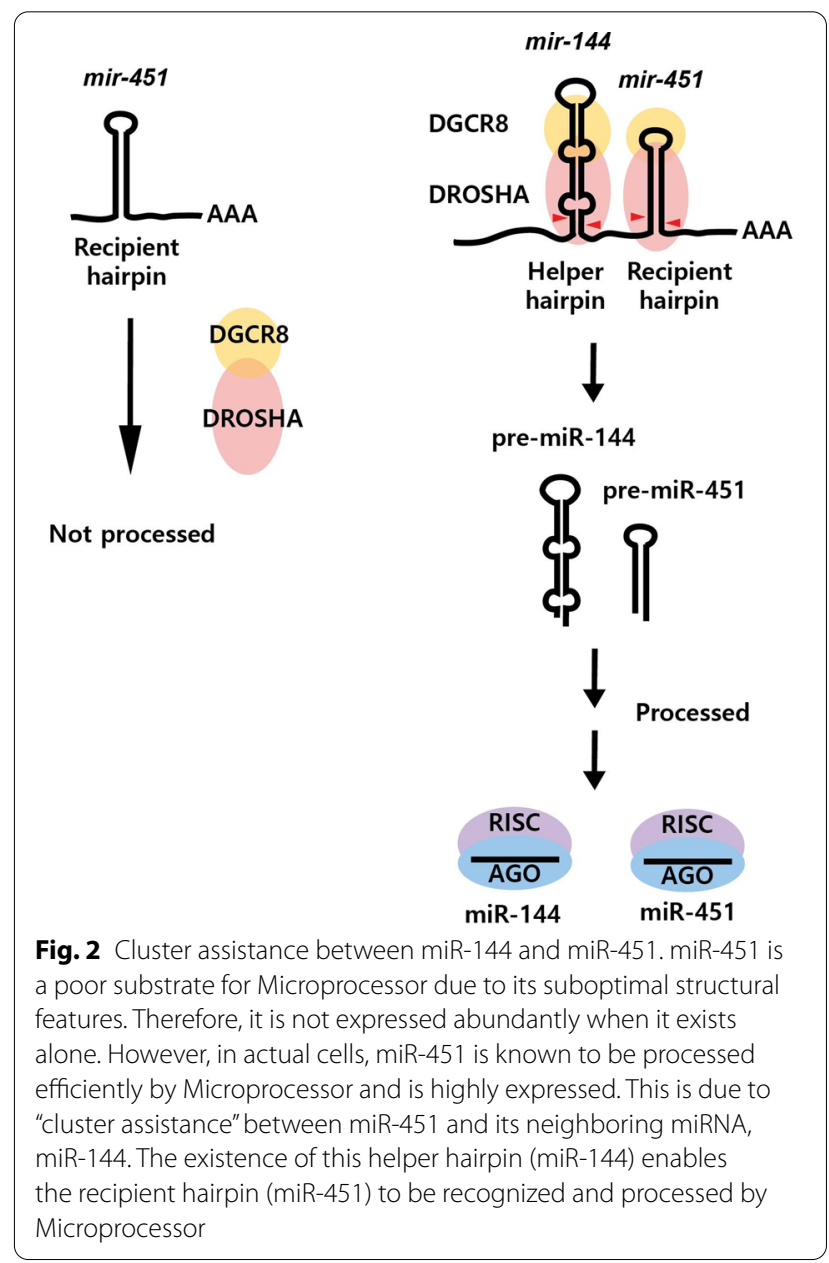


this decreased expression of miR-197a was due to the downregulation of miR-195a [27].

\section{Biogenesis of non-canonical miR-451 is dependent on biogenesis of neighboring miR-144}

The cleavage process of pri-miRNAs directed by the Microprocessor is highly selective. Only very few selected substrates are able to be processed by the Microprocessor and become pre-miRNAs. This selectivity results from the Microprocessor's preference for certain hairpin structures in its substrates. It highly prefers hairpins with a stem length of $35 \pm 1 \mathrm{bp}$, flanking single-stranded regions, with an unstructured terminal loop composed of more than 10 nucleotides, and four specific sequence motifs within the flanking regions and terminal loop [6, 28-32]. These four motifs include the basal UG motif, the apical UGU motif, the flanking CNNc motif, and the mismatched GHG (mGHG) motif [28, 30]. The first three are simple primary-sequence motifs, whereas the last mGHG is a complex primary- and secondary-structural motif [28]. Most of the pri-miRNA hairpins that have been conserved throughout the evolution possess several features of this ideal Microprocessor substrate.

However, there are exceptions and miR-451 is one of those. Its stem is only $31 \mathrm{bp}$ and its apical loop is only 4 nucleotides long [13], making it a very structurally poor Microprocessor substrate. Despite these structural disadvantages, however, miR-451 is still processed by Microprocessor to produce pre-miRNAs and is even one of the most highly expressed miRNAs in erthroblasts and erythrocytes [14]. To understand this abnormal situation, many studies have focused on the biogenesis of miR451 , and recently, it has been suggested that its clusterassisted processing coupled with miR-144 expression is a possible explanation of this abnormality (Fig. 2) [13-16].

miR-144 is located in the same primary transcript as miR-451, but it has structural features that make it a good Microprocessor substrate. It has two motifs and an almost ideal stem-loop structure [13]. However, the expression levels of these two contrasting miRNAs, miR144 and miR-451, are similar and the accumulation of
miR-144 seems to benefit the processing of neighboring miR-451.

To observe cluster assistance in the expression of miR451, miR-451 was expressed in HEK293 cells through a plasmid with a bidirectional promoter. miR-451 was transcribed in one direction, whereas miR-144, which exists in cluster with miR-451, was transcribed in the opposite direction [13]. Thus, miR-451's level of expression increased by around 40-fold when expressed together with miR-144 from the same pri-miRNA transcript, compared to when it was expressed alone. The expression level of miR-144, however, did not change significantly in either situation. Furthermore, when miR-144 was expressed from a different transcript, the benefit it had on miR-451 was not maintained. The benefit remained when the order of miR-451 and miR-144 was switched on the same transcript and also when miR-144 was replaced with another optimal substrate of Microprocessor, miR125a [13]. Lastly, when the hairpin structure of miR-144 was replaced with the hairpin of miR-451, the benefit was lost. These results suggest the existence of "cluster assistance," in which a poor substrate of Microprocessor can be efficiently expressed when clustered on the same transcript with an miRNA that has an optimal hairpin structure [13].

In another study, the expression levels of miR-451 and miR-144 were measured under a number of different circumstances [15]. Experiments such as expressing the two miRNAs from different transcripts, using genetic mutants (deletion of pre-mir-144 or deletion of the terminal loop of pre-mir-144), substituting miR-144 with miR-7a or miR-545, other good substrates of Microprocessor, all showcased similar results as mentioned earlier [29]. In addition, these experiments were performed in vivo using CRISPR/Cas9 technology to overcome the errors of in vitro tests and to confirm that cluster assistance can occur under endogenous environments. The results obtained suggested the existence of cluster assistance in vivo as well as in vitro $[13,15]$.

Studies also suggested that the miR-451 hairpin is not processed efficiently by Microprocessor on its own because of its suboptimal stem-loop structure [13, 15].

Table 1 List of helper and recipient hairpins that exist in clustered orientation, as shown in this review

\begin{tabular}{|c|c|c|c|c|}
\hline & Helper hairpin & Recipient hairpin & Cluster assistance & References \\
\hline 1 & miR-11 & miR-998 & Presence of miR-11 increases the expression level of miR-98 & [20] \\
\hline 2 & miR-BHRF1-2 & miR-BHRF1-3 & $\begin{array}{l}\text { Cluster assistance is valid in certain viral miRNA clusters as well. miR-BHRF1-2 benefits the } \\
\text { processing of miR-BHRF1-3 }\end{array}$ & [24] \\
\hline 3 & miR-195a & miR-497a & Presence of miR-195a increases the expression level of miR-497a & [27] \\
\hline 4 & miR-144 & miR-451 & $\begin{array}{l}\text { Expression of miR-451, the most abundant miRNA in erythrocytes, is highly dependent on } \\
\text { miR-144 }\end{array}$ & [13] \\
\hline 5 & miR-16-1 & miR-15a & miR-16-1 benefits the processing of neighboring miR-15a & [19] \\
\hline
\end{tabular}


When both the stem and the apical loop were lengthened to $35 \mathrm{bp}$ and $12 \mathrm{nt}$, respectively, the ideal length of each feature, the processing rate of miR-451 increased by 170 fold. This observation suggests that unfavorable structural features of miR-451 are the key elements hindering its processing by Microprocessor [13].

\section{Biogenesis of miR-15a is dependent on biogenesis of neighboring miR-16-1}

miR-15a that exists within the miR-15a-16-1 cluster is a recently documented example of cluster assistance. The primary miR-15a hairpin is processed efficiently in the presence of the neighboring optimal hairpin of miR-16-1 [19].

As in miR-451, miR-15a has a suboptimal hairpin structure, which makes it a poor substrate for Microprocessor. Its lower stem has an atypical extended region that has no base pairing, and when one or two pairing point mutations were introduced in this region, miR-15a was expressed at a higher level, even in the absence of the helper hairpin, miR-16-1 $[19,30]$. When this mutation was reversed to mimic the structure of the original lower stem, the expression level of miR-15a was decreased again, demonstrating that this suboptimal stem-loop structure is the reason for its poor expression and its need for cluster assistance [19].
The existence of cluster assistance between miR-15a and miR-16-1 was shown using the GFP and dsRedbased reporter system, which measured the mature miRNA activity of various mutated miRNAs. When the stem-loop structure of miR-16-1 was mutated and destabilized, although the expression level of pri-miRNA did not change substantially, but the mature miR-15a activity decreased. Similar results were also observed under endogenous circumstances, where CRISPR/Cas 9 was used to disrupt the miR-16-1 gene [19]. As with miR-451 and miR-144 $[13,15]$, substituting the assisting miRNA (miR-16-1, in this case) with another optimal miRNA had the same beneficial effects on the processing of miR-15a [19].

Table 1 shows the lists of helper and recipient hairpin pairs that are mentioned in this section.

\section{Characteristics of cluster assistance}

A number of other characteristics of cluster assistance were found through a series of experiments (Fig. 3) [13, 15]. The basal stem of the miR-144 hairpin was mutated to make the structure of the hairpin less optimal as a Microprocessor substrate and to investigate whether the effect of cluster assistance depends on how efficiently Microprocessor recognizes the helper hairpin [13]. As a result, the expression of the recipient gene decreased. Also, when the subsequent mutations were made to

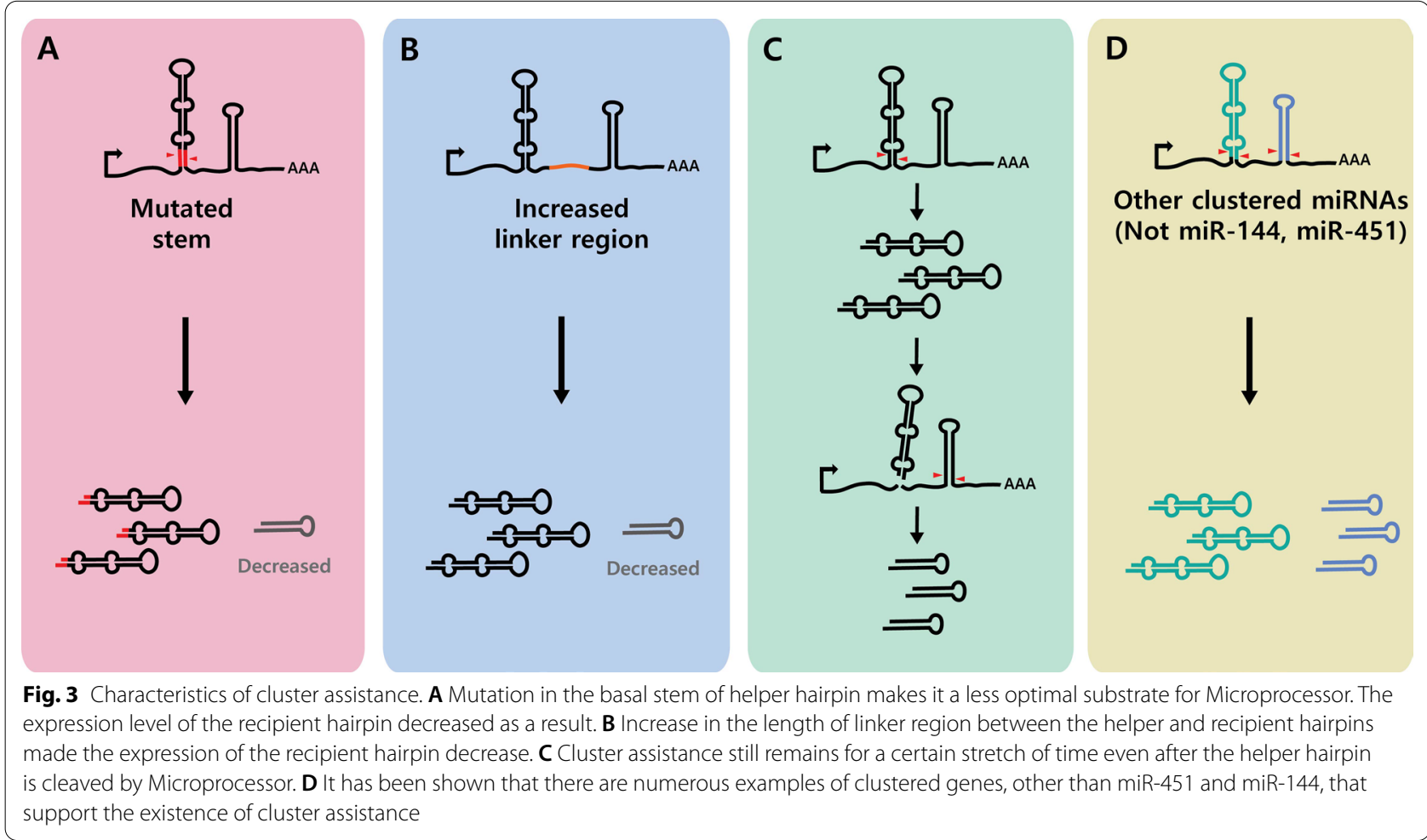


restore the optimal stem length, the level of expression level was restored. These results indicate the existence of correlation between the efficient recognition of the helper hairpin (miR-144) and the expression of the recipient hairpin (miR-451) [13].

To test the possible role of RNAPII in cluster assistance, the miR-144 451 was expressed using a U6 snRNA promoter, which directs RNA polymerase III transcription, instead of RNAPII. Under these conditions, cluster assistance was still evident, indicating that there was no clear effect of RNAPII on cluster assistance and that RNAPII coupling to Microprocessor is not essential to cluster assistance during miR-451 biogenesis $[13,15]$.

The effect of the spacing between the recipient and the helper hairpin on cluster assistance was also tested by increasing the length of the linker region located between miR-144 and miR-451. The results showed that cluster assistance was still evident in the presence of long linkers, but the expression of the miR-451 hairpin clearly decreased as the length between the two miRNAs increased $[13,15]$.

In addition, it has been shown that cluster assistance occurs even after the helper hairpin is cleaved. This situation allows the prolonged association of Microprocessor with its processing miRNAs, thus significantly increases the benefits of cluster assistance [13].

Finally, experiments to determine whether the cluster assistance that enhances the efficiency of suboptimal miRNA biogenesis could be generalized were conducted [15]. By analyzing numerous suboptimal miRNA hairpins with short loops and measuring their relationship with the closest pri-miRNAs, the idea that the interactions between neighboring miRNAs can enhance the biogenesis of suboptimal canonical miRNAs was again supported. However, several individual pri-miRNAs that have short terminal loops but are still expressed efficiently have been discovered. The existence of these miRNAs suggests the possibility that there are different mechanisms that enhance the biogenesis of suboptimal miRNAs when they do not occur within operons [15].

\section{Local recruitment of microprocessor}

Many experimental results, including the experiment in which expression of suboptimal miRNA decreased as the linker region between helper and recipient hairpins increased, suggest the idea that the enhancement of mir451 expression involves local recruitment and transfer of Microprocessor from neighboring optimal miRNAs [13, $15,19]$. The exact mechanism by which the local recruitment of Microprocessor to the helper hairpin enhances the expression of the recipient hairpin is still unknown.

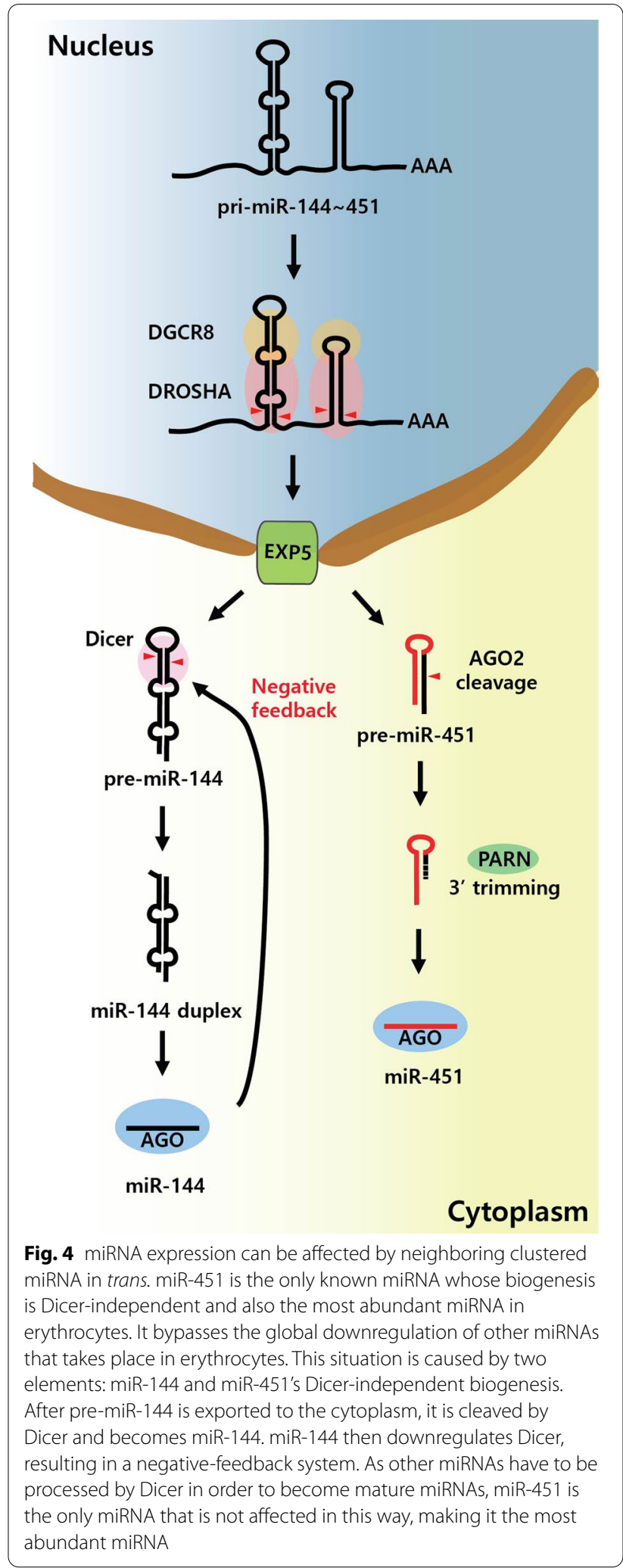


Binding of Microprocessor to the helper miRNA will generally increase the local population of Microprocessors in the neighboring area. However, this cannot be the complete explanation of cluster assistance, as there is no guarantee that an increased population of Microprocessors will result in increased number of Microprocessors binding to the suboptimal hairpin.

Recently, numerous studies have suggested a specific mechanism for this local transfer of Microprocessor [13, $15,19]$. The key elements of the proposed mechanism are the SAFB2 and ERH proteins. It has first been observed that the loss of SAFB2 or ERH results in a significant decrease in the processing of miR-15a ${ }^{\text {mut }}$ (seed mutants of miR-15a), and when SAFB2 and ERH are re-expressed, the normal level of biogenesis was restored. This result indicates that both genes are possible mediators of cluster assistance [19]. It has also been observed that SAFB1, which is highly homologous to SAFB2, can compensate for the loss of SAFB2 to some extent, but not completely [19].

To define how exactly SAFB2 assists biogenesis of miR$15 \mathrm{a}$, and whether it is required for cluster assistance or only for the cleavage of pri-miR-15a after Microprocessor has bound to the miRNA, the seed mutant of miR-15a $\left(\mathrm{miR}-15 \mathrm{a}^{\mathrm{mut}}\right)$ was used. This miR-15a ${ }^{\text {mut }}$ has intrinsic processing activity, and therefore, if SAFB2 is mainly related to cluster assistance, its repression will have effects on miRNA function only when clustering is present. As a result, the loss of SAFB2 and SAFB1 had an impact on miRNA biogenesis only under conditions in which cluster assistance was present, an observation that supports the idea that SAFB proteins are directly involved in the cluster assistance mechanism [19].

In addition, more examples that showcase the involvement of SAFB2 in cluster assistance have been found through experiments. miR-181b in the miR-181a-181b cluster, miR-92a in the miR-17-92 cluster, and miR-425 in the miR-191-425 cluster were shown to be processed by cluster assistance to some extent, and they all had decreased expression when SAFB1 and SAFB2 were absent [19].

A recent study showed the specific interaction between ERH and Microprocessor [33]. The results obtained using cell knockdown, crystal structure and other various methods demonstrate that ERH forms a stable bond in a 2:2 stoichiometry complex with a conserved region in the $\mathrm{N}$-terminus of DGCR8 [33].

As ERH interacts with SAFB1/SAFB2 and also with Microprocessor [33-35], and both SAFB2 and ERH can dimerize, one model of a mechanism for local Microprocessor transfer can be postulated. In this suggested hypothesis, dimerization of SAFB2 and ERH eventually recruits another Microprocessor, which then binds to a nearby recipient hairpin, and enhances its processing [13]. Although this model presently lacks supporting evidence at the moment, the idea that the dimerization of SAFB2 is somehow mandatory for cluster assistance has been suggested by a recent study $[13,15,19]$. This was achieved by shortening the $\mathrm{N}$-terminal of SAFB2 ${ }^{\mathrm{min}}$ (incrementally smaller version of SAFB2), and this experiment demonstrated that the minimal functional region of the SAFB2 protein during cluster assistance consists of a putative coiled-coil domain [19]. As this domain is in charge of the dimerization of SAFB2, it is logical to suggest that the dimerization of SAFB2 is required for cluster assistance.

\section{miRNA expression can be affected by neighboring clustered miRNA in trans}

Recently, data suggesting that neighboring clustered miRNAs regulate the biogenesis of suboptimal miRNA not only in cis, but also in trans have been obtained [16]. An example of this mechanism is the non-canonical, Dicer-independent biogenesis of miR-451.

As mentioned earlier, miR-451 has suboptimal structural features with its short stem and apical loop [6]. Due to its unusually short stem, after being processed by Drosha, pre-miR-451 is not cleaved by Dicer, but instead it goes through a Dicer-independent, non-canonical pathway after it is exported to the cytoplasm. As a result, its second cleavage reaction is performed by $\mathrm{AGO} 2$, which cleaves the middle of the $3^{\prime}$ arm of the hairpin [36, 37]. It is then trimmed by poly(A)-specific ribonuclease (PARN), finally producing mature miR-451 [38]. miR-451 is currently the only known miRNA that is Dicer-independent.

miR-451 is the most highly expressed miRNA in erythrocytes and does not go through the global downregulation of canonical miRNAs that takes place in erythrocytes [14]. A recent study has suggested that this phenomenon could be explained by the trans regulation of miR-144 which is located in the same primary transcript as miR-451 [16].

The efficiency of AGO2 dependent processing and Dicer-dependent processing of pre-miRNAs was compared using Northern blot analysis. It was observed that miR-451 was 7.5-fold more abundant than miR-144 in the peripheral blood of adult fish [16]. However, when the efficiency of AGO2 and Dicer was directly measured by comparing wild-type zebrafish pre-miR-451 (premiR- $451^{\text {Ago2 }}$ ) and pre-miR-451 ${ }^{\text {Dicer, }}$, pre-miR- $451^{\text {Dicer }}$ was more efficiently processed than pre-miR- $451^{\mathrm{Ago} 2}$ by around 20-fold [16]. This result does not agree with the previous result of miR-451 and miR-144 efficiency. This inconsistency can be explained through the analysis of mass spectrometry data of protein abundance during human erythropoiesis in which Dicer shows a steady decline, whereas AGO2 concentration remains the same [39]. Furthermore, three sites that are complementary to 
the miR-144-3p seed sequence were found in the dicer1 3'UTR [16]. This targeting of miR-144 to Dicer was confirmed by reporter assays in zebrafish. These results together suggest a possible role for miR-144 in the downregulation of Dicer during erythropoiesis, forming a negative-feedback loop (Fig. 4) [16].

As PARN trims not only the $3^{\prime}$ end of miR-451, but also numerous other canonical miRNAs [40], decreases in canonical miRNAs due to the repression of Dicer by the negative-feedback of miR-144 allows Dicer-independent miR-451 to be the most abundant miRNA during erythropoiesis, owing to low competition for the final trimming step operated by PARN $[4,38,41]$.

miR-144 thus mediates the repression of Dicer which then eventually allows efficient biogenesis of miR-451 by downregulating other canonical miRNAs. This mechanism is an example of clustered miRNAs regulating each other in trans (Fig. 4) [16].

Understanding the biogenesis of miRNAs will provide better insights into gene regulation and moreover, might even provide invaluable experimental tools. However, there are still more unknown facts than known facts about this mechanism, and numerous studies are being conducted in an attempt to solve these questions. Recent studies suggest a new mechanism in the biogenesis of suboptimal miRNAs $(13,15,16,19)$. Although it has long been known that a large percentage of miRNAs are located in operons, the biological reasons for this genetic composition were not fully understood. However, many recent studies suggest that this cluster of miRNAs assists in the biogenesis of some canonical miRNAs with suboptimal structural features $(13,15$, $16,19)$. More examples of cluster assistance are rapidly being found. Cluster assistance between clustered miRNAs occurs in both cis and trans (16). Cluster assistance provides explanations as to why certain suboptimal miRNAs are expressed at the same levels as, or even higher levels than, their neighboring optimal miRNAs. However, there still remain many blank spaces in the mechanisms of cluster assistance and miRNA regulation in general needs considerable further study. The mechanism by which local recruitment of Microprocessors benefit the recipient hairpins is one of these areas that needs to be studied in more detail (19). Although a large percentage of miRNA genes reside in operons, and many of them are not involved in cluster assistance, cluster assistance will still provide basis for further investigations into the regulation of miRNA.

\section{Acknowledgements}

We apologize to those researchers whose work was not cited in this manuscript due to space limitations.

\section{Authors' contributions}

All authors read and approved the final manuscript.

\section{Funding}

This work was supported by Cooperative Research Program for Agriculture Science and Technology Development (Project No. PJ01577601) Rural Development Administration, Republic of Korea. This work was also supported by the National Research Foundation of Korea (NRF) grant funded by the Korea government (MSIT) (No. 2021R1A5A1032428).

Availability of data and materials

Not applicable.

\section{Declarations}

\section{Competing interests}

The authors declare that they have no competing interests.

\section{Author details}

${ }^{1}$ Department of Applied Biology and Chemistry, Seoul National University, Seoul 08826, Republic of Korea. ${ }^{2}$ Department of Agricultural Biotechnology, Seoul National University, Seoul 08826, Republic of Korea. ${ }^{3}$ Research Institute of Agriculture and Life Sciences, Seoul National University, Seoul 08826, Republic of Korea. ${ }^{4}$ Plant Genomics and Breeding Institute, Seoul National University, Seoul 08826, Republic of Korea. ${ }^{5}$ Research Center for Plant Plasticity, Seoul National University, Seoul 08826, Republic of Korea.

Received: 3 June 2021 Accepted: 7 July 2021

Published online: 15 July 2021

\section{References}

1. Bartel DP (2004) MicroRNAs: genomics, biogenesis, mechanism, and function. Cell 116(2):281-297

2. Pu J, Long Y, Zhou J, Zhan Y, Qin X (2018) MiR-124 regulates apoptosis in hypoxia-induced human brain microvessel endothelial cells through targeting Bim. Appl Biol Chem 61(6):689-696

3. Bartel DP (2018) Metazoan microRNAs. Cell 173(1):20-51

4. Lee Y, Kim M, Han J, Yeom KH, Lee S, Baek SH, Kim VN (2004) MicroRNA genes are transcribed by RNA polymerase II. EMBO J 23(20):4051-4060

5. Nguyen TA, Jo MH, Choi YG, Park J, Kwon SC, Hohng S, Kim VN, Woo JS (2015) Functional anatomy of the human microprocessor. Cell 161(6):1374-1387

6. Han J, Lee Y, Yeom KH, Nam JW, Heo I, Rhee JK, Sohn SY, Cho Y, Zhang BT, Kim VN (2006) Molecular basis for the recognition of primary microRNAs by the Drosha-DGCR8 complex. Cell 125(5):887-901

7. Lee Y, Ahn C, Han J, Choi H, Kim J, Yim J, Lee J, Provost P, Rådmark O, Kim S, Kim VN (2003) The nuclear RNase III Drosha initiates microRNA processing. Nature 425(6956):415-419

8. Zhang H, Kolb FA, Jaskiewicz L, Westhof E, Filipowicz W (2004) Single processing center models for human dicer and bacterial RNase III. Cell 118(1):57-68

9. Yang JS, Lai EC (2011) Alternative miRNA biogenesis pathways and the interpretation of core miRNA pathway mutants. Mol Cell 43(6):892-903

10. Ruby JG, Jan CH, Bartel DP (2007) Intronic microRNA precursors that bypass Drosha processing. Nature 448(7149):83-86

11. Haussecker D, Huang Y, Lau A, Parameswaran P, Fire AZ, Kay MA (2010) Human tRNA-derived small RNAs in the global regulation of RNA silencing. RNA 16(4):673-695

12. Cheloufi S, Dos Santos CO, Chong MM, Hannon GJ (2010) A dicer-independent miRNA biogenesis pathway that requires Ago catalysis. Nature 465(7298):584-589

13. Fang W, Bartel DP (2020) MicroRNA clustering assists processing of suboptimal microRNA hairpins through the action of the ERH protein. Mol Cell 78(2):289-302.e6

14. Zhang L, Flygare J, Wong P, Lim B, Lodish HF (2011) MiR-191 regulates mouse erythroblast enucleation by down-regulating Riok3 and Mxi1. Genes Dev 25(2):119-124

15. Shang R, Baek SC, Kim K, Kim B, Kim VN, Lai EC (2020) Genomic clustering facilitates nuclear processing of suboptimal pri-miRNA loci. Mol Cell 78(2):303-316 
16. Kretov DA, Walawalkar IA, Mora-Martin A, Shafik AM, Moxon S, Cifuentes D (2020) Ago2-dependent processing allows miR-451 to evade the global microRNA turnover elicited during erythropoiesis. Mol Cell 78(2):317-328.e6

17. Altuvia $Y$, Landgraf $P$, Lithwick $G$, Elefant $N$, Pfeffer $S$, Aravin A, Brownstein MJ, Tuschl T, Margalit H (2005) Clustering and conservation patterns of human microRNAs. Nucleic Acids Res 33(8):2697-2706

18. Wang Y, Luo J, Zhang H, Lu J (2016) MicroRNAs in the same clusters evolve to coordinately regulate functionally related genes. Mol Biol Evol 33(9):2232-2247

19. Hutter K, Lohmüller M, Jukic A, Eichin F, Avci S, Labi V, Szabo TG, Hoser SM, Hüttenhofer A, Villunger A, Herzog S (2020) SAFB2 enables the processing of suboptimal stem-loop structures in clustered primary miRNA transcripts. Mol Cell 78(5):876-889

20. Truscott M, Islam AB, Frolov MV (2016) Novel regulation and functional interaction of polycistronic miRNAs. RNA 22(1):129-138

21. Truscott M, Islam AB, Lightfoot J, López-Bigas N, Frolov MV (2014) An intronic microRNA links Rb/E2F and EGFR signaling. PLoS Genetics 10(7):e1004493

22. Truscott M, Islam AB, López-Bigas N, Frolov MV (2011) Mir-11 limits the proapoptotic function of its host gene, dE2f1. Genes Dev 25(17):1820-1834

23. Seto E, Moosmann A, Grömminger S, Walz N, Grundhoff A, Hammerschmidt W (2010) Micro RNAs of Epstein-Barr virus promote cell cycle progression and prevent apoptosis of primary human B cells. PLoS Pathog 6(8):e1001063

24. Feederle R, Haar J, Bernhardt K, Linnstaedt SD, Bannert H, Lips H, Cullen BR, Delecluse HJ (2011) The members of an Epstein-Barr virus microRNA cluster cooperate to transform B lymphocytes. J Virol 85(19):9801-9810

25. Haar J, Contrant M, Bernhardt K, Feederle R, Diederichs S, Pfeffer S, Delecluse HJ (2016) The expression of a viral microRNA is regulated by clustering to allow optimal B cell transformation. Nucleic Acids Res 44(3):1326-1341

26. Porrello ER, Johnson BA, Aurora AB, Simpson E, Nam YJ, Matkovich SJ, Dorn GW, van Rooij E, Olson EN (2011) MiR-15 family regulates postnatal mitotic arrest of cardiomyocytes. Circ Res 109(6):670-679

27. Lataniotis L, Albrecht A, Kok FO, Monfries CAL, Benedetti L, Lawson ND, Hughes SM, Steinhofel K, Mayr M, Zampetaki A (2017) CRISPR/Cas9 editing reveals novel mechanisms of clustered microRNA regulation and function. Sci Rep 7(1):8585

28. Fang W, Bartel DP (2015) The menu of features that define primary microRNAs and enable de novo design of microRNA genes. Mol Cell 60(1):131-145

29. Zeng Y, Yi R, Cullen BR (2005) Recognition and cleavage of primary microRNA precursors by the nuclear processing enzyme Drosha. EMBO J 24(1):138-148
30. Auyeung VC, Ulitsky I, McGeary SE, Bartel DP (2013) Beyond secondary structure: primary-sequence determinants license pri-miRNA hairpins for processing. Cell 152(4):844-858

31. Kwon SC, Baek SC, Choi YG, Yang J, Lee YS, Woo JS, Kim VN (2019) Molecular basis for the single-nucleotide precision of primary microRNA processing. Mol Cell 73(3):505-518

32. Zhang $X$, Zeng $Y(2010)$ The terminal loop region controls microRNA processing by Drosha and Dicer. Nucleic Acids Res 38(21):7689-7697

33. Kwon SC, Jang H, Shen S, Baek SC, Kim K, Yang J, Kim J, Kim JS, Wang S, Shi Y, Li F, Kim VN (2020) ERH facilitates microRNA maturation through the interaction with the N-terminus of DGCR8. Nucleic Acids Res 48(19):11097-11112

34. Drakouli S, Lyberopoulou A, Papathanassiou M, Mylonis I, Georgatsou E (2017) Enhancer of rudimentary homologue interacts with scaffold attachment factor $B$ at the nuclear matrix to regulate SR protein phosphorylation. FEBS J 284(15):2482-2500

35. Kavanaugh G, Zhao R, Guo Y, Mohni KN, Glick G, Lacy ME, Hutson MS, Ascano M, Cortez D (2015) Enhancer of rudimentary homolog affects the replication stress response through regulation of RNA processing. Mol Cell Biol 35(17):2979-2990

36. Yang JS, Maurin T, Robine N, Rasmussen KD, Jeffrey KL, Chandwani R, Papapetrou EP, Sadelain M, O'Carroll D, Lai EC (2010) Conserved vertebrate mir-451 provides a platform for Dicer-independent, Ago2-mediated microRNA biogenesis. Proc Natl Acad Sci USA 107(34):15163-15168

37. Cifuentes D, Xue H, Taylor DW, Patnode H, Mishima Y, Cheloufi S, Ma E, Mane S, Hannon GJ, Lawson ND, Wolfe SA, Giraldez AJ (2010) A novel miRNA processing pathway independent of dicer requires Argonaute2 catalytic activity. Science 328(5986):1694-1698

38. Yoda M, Cifuentes D, Izumi N, Sakaguchi Y, Suzuki T, Giraldez AJ, Tomari Y (2013) Poly(A)-specific ribonuclease mediates 3'-end trimming of Argonaute2-cleaved precursor microRNAs. Cell Rep 5(3):715-726

39. Gautier EF, Ducamp S, Leduc M, Salnot V, Guillonneau F, Dussiot M, Hale J, Giarratana MC, Raimbault A, Luc D, Lacombe C, Mohandas N, Verdier F, Zermati Y, Mayeux P (2016) Comprehensive proteomic analysis of human erythropoiesis. Cell Rep 16(5):1470-1484

40. Lee D, Park D, Park JH, Kim JH, Shin C (2019) Poly(A)-specific ribonuclease sculpts the 3' ends of microRNAs. RNA 25(3):388-405

41. Shukla S, Bjerke GA, Muhlrad D, Yi R, Parker R (2019) The RNase PARN controls the levels of specific miRNAs that contribute to p53 regulation. Mol Cell 73(6):1204-1216

\section{Publisher's Note}

Springer Nature remains neutral with regard to jurisdictional claims in published maps and institutional affiliations.

\section{Submit your manuscript to a SpringerOpen ${ }^{\circ}$ journal and benefit from:}

- Convenient online submission

- Rigorous peer review

- Open access: articles freely available online

- High visibility within the field

Retaining the copyright to your article

Submit your next manuscript at springeropen.com 